\title{
Task Design Principles for Heuristic Refutation in Dynamic Geometry Environments
}

\author{
Kotaro Komatsu $^{1}$ (D) Keith Jones $^{2}$ (D)
}

Received: 27 June 2017 / Accepted: 18 March 2018 / Published online: 7 April 2018

(C) The Author(s) 2018

\begin{abstract}
Task design is increasingly recognised as crucial for enhancing student learning of mathematics. Even so, and despite the significance of mathematical activity related to proofs and counterexamples in school mathematics, the task design principles underpinning students' success in proof-related activity remains under-explored in mathematics education research. What is more, although the affordances of using dynamic geometry environments (DGEs) have been established in the literature, task design in DGEs remains somewhat under-studied. In this paper, we address both these issues by theoretically developing, and empirically testing, task design principles for supporting students' heuristic refutation (revising conjectures/proofs through addressing counterexamples) in DGEs. We use the existing literature to elaborate three design principles: using tasks whose conditions are purposefully implicit, providing tools that enhance the production of counterexamples, and increasing students' recognition of contradictions. To test these principles empirically, we analyse two sets of task-based interviews, one with a triad of secondary school students and the other with a pair of undergraduates, where tasks designed according to the principles were implemented. Our analysis shows that using the tasks enabled the students to engage successfully in heuristic refutation. The matter of examining mathematical definitions when handling counterexamples emerged as an issue for further research.
\end{abstract}

Keywords Counterexample · Definition · Dynamic geometry environment · Proof · Task design principle

Keith Jones

d.k.jones@soton.ac.uk

1 Institute of Education, Shinshu University, Nagano, Japan

2 Southampton Education School, University of Southampton, Southampton SO17 1BJ, UK 


\section{Introduction}

Teachers and researchers in mathematics education have long worked on improving student learning of mathematics. One promising approach to enriching mathematical learning is to enhance task design, because, as ICMI Study 22 confirmed (Watson \& Ohtani, 2015), tasks are the bedrock of day-to-day mathematics lessons and thus have a significant effect on student experience of mathematics and their understanding of the nature of mathematical activity. In the words of Sierpinska (2004, p. 10), "the design, analysis and empirical testing of mathematical tasks, whether for the purposes of research or teaching ... [is] one of the most important responsibilities of mathematics education". In relation to this, Kieran, Doorman and Ohtani (2015) provide a comprehensive review in which they classified task design principles in terms of the scope or level of detail (i.e. grain size); there being grand frames, intermediate-level frames and domain-specific frames. The design principles developed for this paper fall into the last category; the principles are domain-specific with a focus on designing tasks for supporting a particular aspect of student proof-related activity. Below, we describe two points addressed in this paper: heuristic refutation and related task design.

\section{Heuristic Refutation}

Our first focus relates to the mathematical process involving proofs and refutations described by Lakatos (1976). Based on the research by de Villiers (2004, 2010), we use the term heuristic refutation to refer to discovering counterexamples to conjectures/ proofs and revising the conjectures/proofs through addressing the counterexamples. de Villiers (2010) defines heuristic refutation as "reformulating, refining, or polishing a true statement by means of local-counterexamples" (p. 206). We extend this definition to include proofs as the object of revision. We also include counterexamples to conjectures in the means for this revision (with, as discussed later, 'local counterexamples' meaning 'counterexamples to proofs' in Lakatos's terminology). In fact, de Villiers touched on the revision of proofs, and counterexamples to conjectures, in places other than as he defined above.

In our research, the term refutation refers to doubting the truth of conjectures and the validity of proofs. A typical way of refutation is providing a counterexample showing that a conjecture is false but in Lakatos's scheme what is to be refuted includes not only conjectures but also proofs (see the section further below).

Heuristic refutation then means something more than mere refutation, involving not only the discovery of counterexamples but also the revision of conjectures and proofs by means of the counterexamples. Lakatos, in showing how "informal, quasi-empirical, mathematics does not grow through a monotonous increase of the number of indubitably established theorems but through the incessant improvement of guesses by speculation and criticism, by the logic of proofs and refutations" (Lakatos, 1976, p. 5), argued that heuristic refutation plays a crucial role in the development of mathematics.

While, as Hanna (1995) argues, Lakatos's analysis does not capture all the processes that underlie the progress of mathematics, the importance of introducing this view of mathematics into school mathematics has been highlighted by several researchers in mathematics education. For example, Larsen and Zandieh (2008), using Lakatos's ideas to analyse the mathematical activity of a group of undergraduate students, suggested 
that the processes described by Lakatos can be useful for the design of instruction that supports students' reinvention of mathematics. de Villiers (2010), who proposed the notion of heuristic refutation, also concluded that incorporating experimentation and proof in mathematics education can provide students with a deeper, more holistic, insight into the nature of mathematics. In these ways, introducing heuristic refutation into school mathematics enables students to experience an aspect of mathematical practice by which mathematics grows through criticism and improvement.

Despite these important benefits of mathematical activity involving heuristic refutation, task design for facilitating the development of this kind of activity has rarely been explored. Although several studies have explicitly referred to Lakatos's research (e.g. Balacheff, 1991; Komatsu, 2016; Reid, 2002), most of these have focused on how students respond to counterexamples, not on supporting students in generating and addressing counterexamples. A few studies have aimed towards intentionally achieving the mathematical process described by Lakatos during teaching (e.g. Lampert, 1990; Larsen \& Zandieh, 2008), but these studies do not deal with task design. Given the importance of task design, we have worked on this issue by addressing task design in the context of geometry education at the secondary school level (Komatsu, 2017; Komatsu, Jones, Ikeda \& Narazaki, 2017; Komatsu, Tsujiyama, Sakamaki \& Koike, 2014). Our previous studies were conducted in paper-and-pencil environments, and we found that the developed tasks were generally helpful for students' work on heuristic refutation. Nevertheless, we found that some students still encountered difficulties producing diagrams that resulted in the clear refutations of statements or proofs.

\section{Task Design with DGE}

The second focus of this paper, then, is on resolving the above-mentioned student difficulties in producing diagrams by expanding our previous research with paper-andpencil tasks into the use of dynamic geometry environments (DGEs). Many studies have investigated the combination of DGEs and proof-related activity (for extensive review, see Hollebrands, Laborde \& Sträßer, 2008; Sinclair et al., 2016; Sinclair \& Robutti, 2013), with some researchers showing, in particular, how using a DGE to 'drag' on-screen objects to produce a variety of diagrams can allow students to discover refutations of conjectures and proofs (Arzarello, Olivero, Paola \& Robutti, 2002; Healy \& Hoyles, 2001; Marradez \& Gutiérrez, 2000). Nevertheless, although the successful use of DGEs in previous research has been accompanied by carefully designed tasks (Hanna, 2000), how the tasks were designed has often not been fully explained. More research on task design in DGEs is needed; as Sinclair et al. (2016), who reviewed recent studies about DGEs, point out, "[w] hile DGEs have been available for over two decades now, several axes of research remain understudied, particular in terms of task design and teacher practice" (p. 712).

Of course, there have been studies addressing task design in DGEs (e.g. BaccagliniFrank, Antonini, Leung \& Mariotti, 2017; Laborde, 1995; Leung \& Bolite-Frant, 2015; Sinclair, 2003). Indeed, a few researchers have explicated task design principles/models for particular aspects of proof-related activity (e.g. Fahlgren \& Brunström, 2014; Leung, 2011). For example, Prusak, Hershkowitz and Schwarz (2012), in aiming at leading pre-service teachers to engage in productive peer argumentation, proposed three principles for task design: creating a situation of conflict, creating a collaborative 
situation, and providing tools for raising and checking hypotheses. By implementing and analysing a task-based interview, they showed that these principles triggered a shift from visual and intuitive reasoning to logical-deductive reasoning.

While the task design principles elaborated in each of these studies are helpful specifically for the mathematical activity targeted by the respective study, the proposed principles would not be directly applicable to another kind of mathematical process. Our research aims at the development of a process involving heuristic refutation. Given that counterexamples play a crucial role in eliciting this process, task design that allows the occurrence of counterexamples is necessary for our aim. This kind of task design principle, however, is not seen among the principles formulated in the existing studies. Hence, it is necessary to create a fresh set of task design principles specifically aimed at helping students to engage in heuristic refutation.

\section{Research Question}

Based on the above discussion, in this paper we address the following research question: What principles can underpin the design of DGE tasks that support student activity related to heuristic refutation? To explore this question, we theoretically develop task design principles based on the existing literature and then empirically test these principles by analysing task-based interviews with a triad of secondary school students and a pair of undergraduates. We begin by examining the meaning and nature of proofs and refutations, and how heuristic refutation, the focus of this paper, corresponds to refuting and the transition between conjecturing/proving and refuting.

\section{The Meaning and Nature of Proofs and Refutations}

The mathematical process that Lakatos described in his book Proofs and Refutations (Lakatos, 1976) can be depicted as in Fig. 1. One makes conjectures (or is provided with statements) and then attempts to prove them. In doing so, one is confronted with refutations of the conjectures/statements and/or proofs, and the conjectures/statements and/or proofs are revised through addressing the refutations (Komatsu, 2016). Heuristic refutation, which is the focus of this paper, corresponds to refuting and the transition between conjecturing/proving and refuting in Fig. 1.

At this point, we clarify three points regarding Fig. 1. First, as mentioned above, some activity begins with making conjectures, and other activity with considering

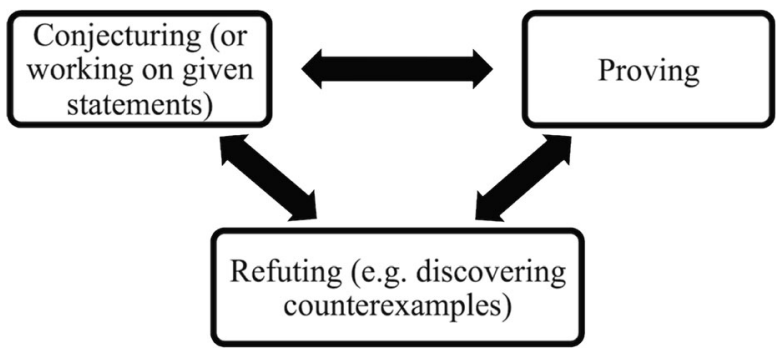

Fig. 1 Mathematical activity involving proofs and refutations 
statements given by others. This is the case in Lakatos's book Proofs and Refutations where he started his account by introducing the Descartes-Euler conjecture on polyhedra as a given statement. Polya (1954) had already elaborated how this conjecture could be produced, and Lakatos mentioned that his own story was a sequel to Polya's description.

Second, each of the three elements in Fig. 1 includes not only final products (e.g. proofs) but also processes where one attempts to create the products (e.g. planning proofs). Here, we interpret the term proof in a broad sense, such that we include informal forms of proofs such as generic proofs; that is, proofs carried out on generic examples (e.g. Leron \& Zaslavsky, 2013). We also include deductive proofs that are valid only for subsets of all cases considered in conjectures and statements. One possibility is that students intentionally select a subset of the whole family in order to get an idea of how to start proof construction. Another possibility is that students intend to produce a general proof but inadvertently use some property specific to a subset, and thus, their proof is only partially general (this occurred in our empirical study that we present later in this paper). These two possibilities are likely to happen as interim stages in the process of proof construction. Our broader conception of proof is epistemologically consistent with Lakatos's $(1976,1978)$ view of proof because he dealt with informal proofs (e.g. Cauchy's proof for the Descartes-Euler conjecture, where a polyhedron is imagined to be made of thin rubber, one of the faces of the polyhedron is cut out, and so on), and with proofs that were deductive but only partially general (e.g. Gergonne's proof and Legendre's proof, both of which are not as general as Cauchy's proof).

Third, although the word refutation is sometimes used only in relation to conjectures and statements, not for proofs, this study utilises it for both conjectures/statements and for proofs. This is also consistent with Lakatos's conception because he considered that proof was inextricably linked to refutations (Balacheff, 1991; Reid, Knipping \& Crosby, 2008). In particular, he coined the term local counterexample to refer to a case that rejects a step in a proof (and global counterexamples for the refutations of conjectures).

\section{Towards a Set of Task Design Principles}

As noted above, some studies have worked on task design and classroom intervention relevant to conjecturing, the transition from conjecturing to proving, and proving, as depicted in Fig. 1 (e.g. the special issue of Educational Studies in Mathematics published as vol. 96, issue 2). An example is Lin, Yang, Lee, Tabach and Stylianides (2012) who elaborated a set of task design principles for engaging students in activities related to conjecturing and proving. In contrast, our study focuses on refuting and the transition between conjecturing/proving and refuting, as in Fig. 1. This focus means that the task design principles developed in this paper are for students who are capable of proof construction, rather than for all students.

Despite this restriction on the scope of the target students, we contend that our principles are valuable because even high-attaining students might not possess a welldeveloped view on proving. For example, Healy and Hoyles (2000), in a survey involving high-attaining students (aged 14-15) in England and Wales, found that many 
students, when asked to choose arguments they would adopt for themselves and those that they considered would receive the best mark from their teachers, selected different arguments. In particular, the students thought that their teachers would most highly value an argument that was seemingly deductive but, in fact, consisted of meaningless algebraic expressions. This result shows that even high-attaining students regard proving activities as mere rituals in schools, rather than as essential activities for deepening their mathematical understanding and enriching their mathematical practice.

Our task design principles would be helpful for addressing such students' formulaic views of mathematics, because the principles aim to engage students in heuristic refutation through which they could experience a dynamic process of mathematics where conjectures and proofs progress through forms of criticism and improvement. As de Villiers (2010) pointed out, this kind of experience could form a foundation for students to gain authentic insight into the nature of mathematics (as we set out in the 'Introduction').

We undertook a thorough review of existing literature and, based on this, identified three promising avenues towards our task design principles to support student activity in heuristic refutation. First, as Hanna (1995) noted, Lakatos's case for heuristic proof analysis as a general method rests on "its successful use in the study of polyhedra, an area in which it is relatively easy to suggest the counterexamples which this method requires" (p. 45). In other words, it is necessary to create tasks intentionally where specific proofs can be constructed and counterexamples can be produced. In Lakatos's story, it was the ambiguous meaning of 'polyhedra' that played a role in triggering the production of such proofs and counterexamples; the (imaginary) teacher and students shared a specific proof based on a single case (cube) as a generic example, and then considered various objects, such as a pair of polyhedra with an edge in common, or polyhedra with holes (i.e. the picture-frame type). The students regarded these objects as polyhedra and proposed them as counterexamples. Hence, in the same way as in Lakatos's research, it is important to develop tasks whose conditions are purposefully implicit so that students can avail themselves of, and consider, particular proofs and counterexamples. This is supported by our previous studies demonstrating that specific tasks, in the form of proof problems with diagrams that include hidden conditions, are helpful for students to explore proofs and counterexamples in secondary school geometry (Komatsu, 2017; Komatsu et al., 2017).

Second, a number of studies have reported that students encounter difficulties in producing appropriate counterexamples, irrespective of whether the abovementioned implicitness of task conditions is involved (e.g. Hoyles \& Küchemann, 2002; Ko \& Knuth, 2013; Komatsu et al., 2017). Thus, it is important to prepare tools that help students produce counterexamples. DGEs have the potential to play such a role in geometry education because each type of DGE has the facility of making it straightforward to transform diagrams by dragging points or lines - thereby giving students easy access to a variety of diagrams (Arzarello et al., 2002; Baccaglini-Frank \& Mariotti, 2010; Marradez \& Gutiérrez, 2000). This argument is supported by Healy and Hoyles (2001) and Marradez and Gutiérrez (2000) who reported examples of students using DGEs to produce counterexamples.

Researchers have classified dragging modalities as well as measuring modalities in DGEs, including wandering dragging, dummy locus dragging, wandering measuring, guided measuring, and so on (e.g. Arzarello et al., 2002; Olivero \& Robutti, 2007). On the one hand, these classifications show the power of DGEs to enhance the production of counterexamples because some of the modalities are particularly relevant to the 
refutations of conjectures and proofs: viz. dragging test, validation measuring and proof measuring. On the other hand, different dragging modalities may lead to different results in generating diagrams, and certain dragging modalities (e.g. wandering dragging, which is performed casually, without any goal) may not be helpful for finding refutations. Hence, task design needs to include the purpose of the use of the DGE in tasks. An example would be a sentence in a task stating 'use a DGE to investigate whether there are counterexamples' rather than simply 'use a DGE'.

Third, research indicates that when students encounter counterexamples, some may merely reject the counterexamples and not attempt to revise their conjectures (Balacheff, 1991; Zazkis \& Chernoff, 2008). To address this issue, we focus on the potential of the 'confusion' generated by contradictions; confusion that can be beneficial for learning when appropriately induced (D’Mello, Lehman, Pekrun \& Graesser, 2014). Mathematics education researchers have reported that proof-related activity can be purposefully stimulated in students when the students face contradictions between their expectations and the results they obtain (Prusak et al., 2012; Zaslavsky 2005). To evoke such contradictions, an approach likely to be helpful is to sequence multiple tasks intentionally (rather than use a single task) so that contradictions can arise among the tasks (Hadas, Hershkowitz \& Schwarz, 2000). For instance, Prusak et al. (2012) conducted a task-based interview with a pair of pre-service teachers where they used a set of three tasks wherein the first two were designed to elicit intuitive thinking, an approach that was not applicable to the third task. They reported that the pre-service teachers experienced a contradiction between their convictions based on intuitive thinking after the first two tasks and the failure of this approach in the third task, and that this experience helped them solve the third task through logical-geometrical reasoning.

In summary, we propose the formulation of three task-design principles for supporting student activity related to heuristic refutation, as seen in Table 1. Roughly speaking, Principles 1, 2, and 3 correspond respectively to the transition from conjecturing/proving to refuting, refuting, and the transition from refuting to conjecturing/proving in Fig. 1.

Within these design principles, the meaning of counterexample needs clarification: we use this term in a subjective way, where we regard a case as a counterexample if students consider that the conjecture/statement or proof is refuted by the case, regardless of whether it fully constitutes a counterexample in a mathematical sense. In particular, if task conditions are not clear, it can be a subtle matter to discern whether a case is a counterexample or a non-example (see Table 2 for an example of the distinction between counter-example and non-example). Thus, in this study, we interpret the meaning of 'counterexample' from a subjective, student standpoint, not from a fully mathematical standpoint.

Table 1 Task design principles for heuristic refutation

\begin{tabular}{ll}
\hline Principle & Description of the principle \\
\hline Principle 1 & $\begin{array}{l}\text { Using tasks whose conditions are purposefully implicit and thus allow the production } \\
\text { of particular proofs and the occurrence of counterexamples. }\end{array}$ \\
Principle 2 & $\begin{array}{l}\text { Providing tools that enhance the production of counterexamples, while making explicit } \\
\text { the purpose of the tools' use (i.e. investigating the existence of counterexamples). }\end{array}$ \\
Principle 3 & $\begin{array}{l}\text { Increasing students' recognition of contradictions that can help them revise } \\
\text { conjectures/statements and/or proofs. }\end{array}$
\end{tabular}


Table 2 Relationship between the task design principles and the designed tasks

\begin{tabular}{|c|c|}
\hline Principle & Task design \\
\hline Principle 1 & $\begin{array}{l}\text { - Task 1-1. The condition of the task is not clearly presented in terms of the locations o } \\
\text { points A-D. A proof of a conjecture (e.g. } \triangle \mathrm{PAB} \sim \triangle \mathrm{PDC} \text { ) can be constructed for the } \\
\text { given diagram. However, if the locations of these points are changed, local } \\
\text { counterexamples to the proof can be discovered. } \\
\text { - Task 2-1. The task includes a hidden condition in the given diagram assuming that } \\
\text { perpendicular lines from points A and } \mathrm{C} \text { always intersect with diagonal BD. A proof } \\
\text { of the statement can be constructed for the given diagram. However, if the shape } \\
\text { of parallelogram ABCD is changed, cases where quadrilateral AECF is not } \\
\text { constructed can be discovered (in a mathematical sense, this is a non-example } \\
\left.\text { rather than a counterexample }{ }^{\mathrm{a}}\right) \text {. }\end{array}$ \\
\hline Principle 2 & $\begin{array}{l}\text { - Task 2-2 (similarly for Task 1-2). Students are asked to use a DGE to construct the } \\
\text { diagram given in Task 2-1 (similarly for Task 1-1), to transform it as required by } \\
\text { dragging, and to investigate the truth of the statement (or the truth of students' } \\
\text { conjecture and the validity of their proof in Task } 1-2 \text { ). }\end{array}$ \\
\hline Principle 3 & $\begin{array}{l}\text { The sequence of Tasks } 2-1 \text { and } 2-2 \text { (similarly for Tasks } 1-1 \text { and } 1-2 \text { ). It is entirely } \\
\text { possible for students to be invited to begin producing various diagrams with a } \\
\text { DGE prior to proof construction; however, without proving the statement, students } \\
\text { may not be convinced of its truth and, consequently, may not feel a contradiction } \\
\text { when facing the refutation of the statement. To avoid this, we sequenced Tasks } 2-1 \\
\text { and } 2-2 \text { (similarly for Tasks } 1-1 \text { and } 1-2 \text { ) such that students are asked to construct } \\
\text { a proof and then transform the diagram with the DGE. The expectation is that } \\
\text { proof construction in Task } 2-1 \text { (similarly for Task } 1-1 \text { ) could increase students' } \\
\text { conviction of the truth of the statement, and that thereby, a contradiction could } \\
\text { likely be evoked more sharply between the students' conviction and its subsequent } \\
\text { refutation in Task } 2-2 \text { (similarly for Task } 1-2 \text { ). }\end{array}$ \\
\hline
\end{tabular}

\footnotetext{
${ }^{a}$ The statement in Task 2-1 assumes (implicitly) that it is possible to construct quadrilateral AECF, and under this assumption, infers that the quadrilateral AECF is a parallelogram. Because the case mentioned here does not satisfy this assumption, it is, mathematically, a non-example rather than a counterexample
}

\section{Methods}

\section{Tasks}

To address the research question adopted by our study, the principles summarised in Table 1 were used to design three sets of tasks for participants, two of which (see Fig. 2) were implemented through task-based interviews. Table 2 explains the relationship between the task design principles and the designed tasks. In the descriptions of the second and third principles in the table, we focus on Task 2 (and say similarly for Task 1) because the ways in which our task design is based on these principles are almost identical between the two tasks. ${ }^{1}$

There is more we need to say about the designed tasks in terms of counterexamples. We take Task 1 as an example. Although Task 1-1 includes one diagram where points A-D are located in certain places on the circle, the problem sentences per se make no reference to the specificity of the locations of these four points. Thus, this task, on the one hand, requires making and proving a conjecture while taking into account not only

\footnotetext{
${ }^{1}$ When we mention 'Task 2', we include both Tasks 2-1 and 2-2; 'Task 1' has corresponding coverage.
} 


Task 1-1. (1) As shown in the diagram given, there are
four points $\mathrm{A}, \mathrm{B}, \mathrm{C}$, and $\mathrm{D}$ on circle $\mathrm{O}$. Draw lines $\mathrm{AC}$
and $\mathrm{BD}$, and let point $\mathrm{P}$ be the intersection point of the
lines. What relationship holds between $\triangle \mathrm{PAB}$ and
$\Delta \mathrm{PDC}$ ? Write your conjecture. (2) Prove your
conjecture.
Task 1-2. Construct the diagram shown in Task 1-1 with
a DGE. Move points A, B, C, and D on circle O to
examine the following questions. (1) Is your conjecture
in Task 1-1 always true? (2) Is your proof in Task 1-1
always valid?
Task 2-1. In parallelogram ABCD, we draw
perpendicular lines AE and CF to diagonal BD from
points A and C, respectively. Prove that quadrilateral
AECF is a parallelogram.
Task 2-2. Construct the diagram shown in Task 2-1
using a DGE. Move the vertices to change the shape of
parallelogram ABCD, and examine whether
quadrilateral AECF is always a parallelogram.

Fig. 2 Tasks used in the task-based interviews

a particular case represented by the given diagram, but also a general domain where points A-D are located in various orders and places on the circle.

On the other hand, one diagram inevitably has to be one particular case, and it is likely that a conjecture/proof based on the diagram is true/valid only for a subset of the whole family. For example, in Task 1-1, suppose that students conjecture that $\triangle \mathrm{PAB} \sim$ $\triangle \mathrm{PDC}$, and they prove it by showing that $\angle \mathrm{APB}=\angle \mathrm{DPC}$ (using the equality of vertical angles) and $\angle \mathrm{PAB}=\angle \mathrm{PDC}$ (using the inscribed angle theorem). This proof, however, becomes invalid in other subsets of the general domain: the inscribed angle theorem cannot be used for showing $\angle \mathrm{PAB}=\angle \mathrm{PDC}$ in Fig. 3e shown later. This case is a local counterexample to the proof in Lakatos's (1976) terminology. It is thus necessary to revise the proof by using a different theorem. The conjecture $(\triangle \mathrm{PAB} \sim$ $\triangle \mathrm{PDC}$ ) also cannot be considered for some cases of the general domain (e.g. Fig. 3a). To actualise the first design principle (Table 1), we capitalise on this type of task where the task conditions are implicit due to the particularity of diagrams; thus, particular proofs and counterexamples can occur.

Tasks 1 and 2 were designed to have two salient differences between them. The first difference corresponds to conjecturing (or working on given statements) in the framework shown in Fig. 1: in Task 1-1, students are invited to make a conjecture, and, in Task 2-1, are given a statement. The second difference relates to the activity subsequent to refuting in Fig. 1, specifically to what students are expected to revise in their responses to Tasks 1-2 and 2-2. In Task 2, there is a case where quadrilateral AECF, mentioned in the conclusion in Task 2-1, cannot be constructed. To include this case, it is, on the one hand, necessary to revise the statement in Task 2-1. On the other hand, when the positions of points A-D are changed in Task 1-2, the triangles mentioned in Task 1-1 can still be constructed (except in a few special cases; see Fig. 3). However, as mentioned above, it is still necessary to revise the proof constructed in Task 1-1.

For each task-based interview, we prepared two worksheets, one of which included only Task 1-1 (or Task 2-1) and another one of which included Task 1-2 (or Task 2-2). Our research design entailed beginning by giving out the first worksheet, and then to provide the second worksheet after the participants had solved the task in the first worksheet. 


\section{Participants}

We conducted three task-based interviews (in each of which students attempted one of the tasks described above) in order to investigate how the tasks facilitated students' process of conducting heuristic refutation. The first author of this paper carried out all of the task-based interviews during which the DGE GeoGebra (in Japanese) was used.

Task 1 was implemented with a triad of students in an upper secondary school affiliated with a Japanese national university. We recruited participants from among 11th graders (16-17 years old) in the school who were taking a mathematics subject; the three participants voluntarily took part in the task-based interview. Their teacher judged that their mathematical capabilities were above average. The students were familiar with constructing geometric proofs using the conditions for congruent triangles and those for similar triangles. They were also familiar with the theorems relevant to Task 1, namely the inscribed angle theorem, the inscribed quadrilateral theorem, and the alternate segment theorem.

Given that the students had never experienced a DGE, we devoted $4 \mathrm{~h}$ before the task-based interview to introducing DGE for basic plane geometry. For example, after teaching basic constructions (e.g. the perpendicular bisector of a segment), dragging, and measuring, we asked the students to construct rectangles that could be preserved when points or lines were dragged. We did this in order to provide the students with an opportunity to experience what is commonly called the 'dragging test' (Arzarello et al., 2002). We also gave a problem where the students were invited to make and prove a conjecture.

Task 2 was implemented separately with two pairs of undergraduate students (four students) enrolled in the mathematics education course in the Faculty of Education of a Japanese national university. Each of the students was a prospective teacher at either the primary school level or the secondary school level. We chose undergraduates as participants because proof construction in Task 2-1 might be challenging for secondary school students. The undergraduates were personally invited to take part in the task-based interviews and were selected on the basis of our inkling that they would likely provide expansive verbal discussion useful for data analysis. Because the undergraduates already had considerable experience in using (such as GeoGebra, Cabri and Cabri 3D), we did not allocate specific time for them to introduce the DGE before the task-based interviews.

\section{Data Collection and Analysis}

In each of the task-based interviews, the students were asked to solve the tasks collaboratively with a pen and a computer. Each task-based interview lasted for approximately $35 \mathrm{~min}$. The task-based interviews were video-recorded and audiotranscribed; in each, two cameras were used for recording, one placed to film the students and the other to record the computer screen. The worksheets the students completed, and the DGE files they made, were collected.

These data were analysed in three phases. In phase 1, the whole process of each task-based interview was divided into segments on the basis of the three elements in the theoretical framework shown in Fig. 1 (i.e. conjecturing, proving and 


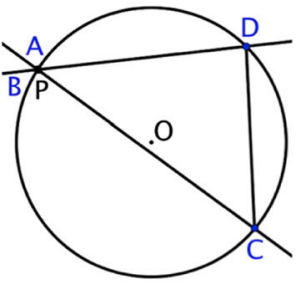

a

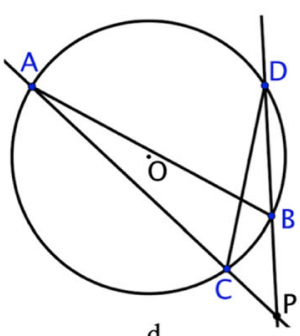

d

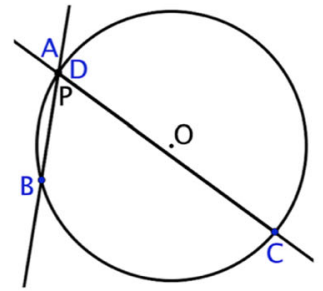

b

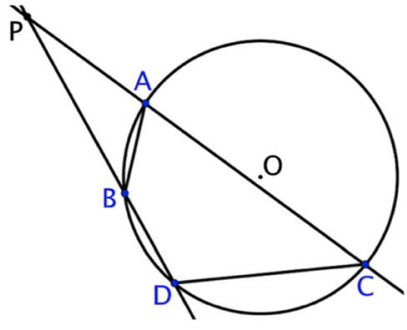

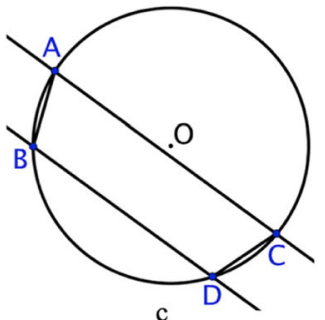

C

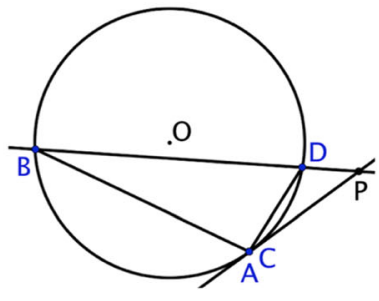

f

Fig. 3 The types of diagrams produced by the students in Task 1-2

refuting). In phase 2, we focused on the refuting element of the framework and classified the diagrams the students produced when they tackled Tasks 1-2 and 22. In phase 3 , we analysed how the students dealt with the diagrams they produced; for example, whether the students accepted the diagrams as showing counterexamples and whether and how they revised their conjecture, the given statement, and/or their proofs.

In the next two sections, we present the results of our analysis in the form of two cases. First, we present an account of the task-based interview with the triad of secondary school students (Kakeru, Sakura, and Yuka; pseudonyms) who tackled Task 1. Then, for Task 2, because the two pairs of undergraduate students followed almost identical paths, this paper focuses on only one pair (Kenta and Tsubasa; pseudonyms) to avoid repetition. For readability, we present our analysis of the effects of the implemented tasks in the last subsection for each case (rather than in the general 'Discussion' section), as this allows us to reduce the distance in the text between the results of each task-based interview and the analysis of the effects of using the tasks. English translations of the students' and interviewers' utterances, and of the students' proofs, are given from the original Japanese.

\section{Case 1: Kakeru, Sakura and Yuka Tackle Task 1}

\section{The Conjecture, Proof, and Types of Diagrams Produced by the Students}

When Kakeru read the problem sentences in Task 1-1, Sakura immediately responded with the conjecture that " $\triangle \triangle \mathrm{PAB}$ and $\triangle \mathrm{PDC}$ are $]$ similar?" The students then together constructed the following proof on their worksheet: 
In $\triangle \mathrm{PAB}$ and $\triangle \mathrm{PDC}$,

From the vertical angles, $\angle \mathrm{APB}=\angle \mathrm{DPC}(1)$

From arc $\mathrm{BC}$, since inscribed angles are equal, $\angle \mathrm{PAB}=\angle \mathrm{PDC}(2)$

From (1) and (2), since two pairs of angles are equal, $\triangle \mathrm{PAB} \sim \triangle \mathrm{PDC}$.

Afterwards, the students tackled Task 1-2 with the DGE. As they dragged points A-D, they produced the six types of diagrams shown in Fig. 3. In Fig. $3 \mathrm{a}^{2}$ either $\triangle \mathrm{PAB}$ or $\triangle \mathrm{PDC}$ is not constructed. In cases like Fig. 3b, c, neither triangle is made (in Fig. 3c, lines $\mathrm{AC}$ and $\mathrm{BD}$ are parallel, and therefore, point $\mathrm{P}$ is not constructed). The students considered the three types of diagram (illustrated by Fig. $3 \mathrm{a}-\mathrm{c}$ ) to be counterexamples to their conjecture.

While in Fig. 3d, e the point $\mathrm{P}$ is located outside circle $\mathrm{O}$, in Fig. 3f, the students considered points $\mathrm{A}$ and $\mathrm{C}$ (or points $\mathrm{B}$ and $\mathrm{D}$ ) to coincide, and regarded line $\mathrm{AC}$ (or line $\mathrm{BD}$ ) to be a tangent to circle $\mathrm{O} .{ }^{3}$ In this paper, we examine the cases of Fig. 3e, f because the students devoted more effort to these ones compared to the others.

\section{The Case Where Point P Is Outside the Circle}

The three students produced Fig. 3e at the beginning of their work on Task 1-2 and discussed it as follows:

116 Kakeru: Is the conjecture in the question 1 [Task 1-1], similarity, always true? [reading the problem sentence.]

117 Sakura: Not similar.

118 Yuka: In this case, ... impossible.

119 Kakeru: The intersection point is outside the circle.

This verbal exchange shows that Sakura and Yuka were confronted with a contradiction between Task 1-1 and Task 1-2. In Task 1-1, the three students made a conjecture and proved it. In Task 1-2, they encountered Fig. 3e, which they regarded as a counterexample to their conjecture (lines 117 and 118). This contradiction then triggered a debate between Sakura and Yuka, on the one side, and Kakeru on the other. The focus of their discussion is evident in the following exchange:

\footnotetext{
${ }^{2}$ It is more exact to state 'the type illustrated in Figure X', not just 'Figure X', because the students dragged points to consider more than one diagram for one type. However, hereafter we simply state 'Figure $\mathrm{X}$ ' for readability.

${ }^{3}$ Note that, strictly speaking, this type is different from the original problem where, for example, line AC cannot be drawn if points $\mathrm{A}$ and $\mathrm{C}$ coincide.
} 
132 Kakeru: We can say that they are similar.

133 Sakura: Why? We can't say that.

134 Kakeru: Because.

135 Sakura: Wait. Because.

136 Kakeru: This $[\angle \mathrm{P}]$ is equal.

137 Sakura: [Triangle] PAB.

138 Kakeru: [Triangles] PAB and PDC. These are similar. This and this $[\angle \mathrm{P}]$ are common and equal. Then, because [quadrilateral $\mathrm{ABDC}$ is] a quadrilateral that is inscribed to the circle.

139 Sakura: That one.

140 Kakeru: This $[\angle \mathrm{PAB}]$ and this $[\angle \mathrm{PDC}]$ are equal.

In this exchange, Kakeru claimed that their conjecture was still true (line 132), but Sakura challenged him (lines 133 and 135). In response to the challenge, Kakeru mentioned that $\angle \mathrm{APB}$ and $\angle \mathrm{DPC}$ were common and thus equal, and then proposed using the inscribed quadrilateral theorem to show that $\angle \mathrm{PAB}=\angle \mathrm{PDC}$ (lines 138 and 140); this theorem expresses that an interior angle of a cyclic quadrilateral is equivalent to the exterior angle of the opposite angle. Sakura agreed with Kakeru's proposal (line 139), and thus, the students were able to resolve the debate by showing that Fig. $3 \mathrm{e}$ is actually not a counterexample to their conjecture (i.e. $\triangle \mathrm{PAB}$ and $\triangle \mathrm{PDC}$ are still similar in this case).

\section{The Case Where a Line is a Tangent to the Circle}

After resolving their debate about the case of Fig. 3e, the students then examined the case of Fig. 3f. Here, Kakeru was confident that their conjecture would still hold with this type and suggested using the alternate segment theorem to prove the conjecture in this case; this theorem expresses that the angle between a tangent and a chord at the point of contact is equal to the angle in the alternate segment. However, when he began explaining his idea to Sakura and Yuka, a doubt entered his mind regarding why line AP could be regarded as a tangent here. Although the students struggled to resolve this doubt, they continued to anticipate that their conjecture would be preserved in this type. Eventually, they assumed that line AP was the tangent by measuring $\angle \mathrm{PAO}$ and finding that it was almost $90^{\circ}$. The subsequent interaction between the students was as follows:

357 Kakeru: If we consider this as a tangent, we can use the theorem about the angle formed by a tangent and a chord.

358 Sakura: I see. 
359 Kakeru: We can show the similarity.

360 Sakura: This $[\angle \mathrm{DCP}]$ and this $[\angle \mathrm{PBA}]$ and $\mathrm{P}$.

361 Kakeru: Yes. This and this $[\angle \mathrm{DCP}=\angle \mathrm{PBA}]$ and $\mathrm{P}$ are equal.

In this exchange, Kakeru and Sakura used the alternate segment theorem to show that $\angle \mathrm{PBA}=\angle \mathrm{PCD}$ (lines 357, 360, and 361). The students also noted that $\angle \mathrm{APB}=\angle \mathrm{DPC}$ (lines 360 and 361). Although they did not specify any reason for this equality, they derived it from the identity of these angles. Thus, they were able to prove their conjecture $(\triangle \mathrm{PAB} \sim \triangle \mathrm{PDC})$ for Fig. 3f, assuming, without proof, that line $\mathrm{PA}$ is tangent to circle $\mathrm{O}$.

\section{Examination and Revision of the Initial Proof}

Task 1-2, shown in Fig. 2, consisted of (1) examination of a conjecture and (2) examination of a proof. In fact, however, the students concentrated on (1), and finished their work without considering (2): Kakeru and Yuka concluded their activity by saying, "The conclusion does not necessarily hold [as Figs. 3a-c exist]" (italics used to show emphasis). Hence, the interviewer asked them, "Please read again the sentences in Task 2 , carefully. When you say 'does not hold', do you mean your conjecture is false, or your proof is invalid?" When working on this question, the students noticed that their initial proof in Task 1-1 had become invalid in the diagrams they had produced. This is illustrated in the exchange below, where they discussed the case of Fig. 3e:

456 Sakura: We wrote, "From arc $\mathrm{BC}$, since inscribed angles are equal, $\angle \mathrm{PAB}=$ $\angle \mathrm{PDC}$ ".

457 Kakeru: [Angles] PAB and PDC. This [the initial proof] is for this case [shown in Task 1-1 in Fig. 2].

458 Sakura: This [the last line in the proof] is valid, but the sentences [the second and third lines in the proof] are not valid, right?

459 Kakeru: Yes. Because.

460 Yuka: This [the initial proof] is only for this [the diagram shown in Task 1-1 in Fig. 2].

461 Kakeru: This is only for this [repetition of Yuka's remark].

462 Yuka: If so, this proof ....

463 Sakura: Is not always valid, right?

Sakura pointed out that the reasons given in the students' initial proof (namely, the equality of vertical angles and the inscribed angle theorem) were not applicable to 
Fig. 3e (line 458). In Lakatos's (1976) terminology, she found that this case was a local counterexample to their proof (line 463). The students therefore revised the proof, by changing their reasons, pointing instead to the identity of the angles and the inscribed quadrilateral theorem. Afterwards, they examined the case of Fig. $3 \mathrm{f}$ as well, and revised the initial proof in a similar way, referring to the alternate segment theorem.

\section{Analysis of Case 1}

The students in case 1 were able to engage in the process depicted in Fig. 1. After making and proving a conjecture in Task 1-1, they produced diagrams to inspect whether their conjecture was always true in Task 1-2. Sakura and Yuka first considered Fig. $3 \mathrm{e}$ to be a counterexample to their conjecture. However, the students overcame this problematic situation by proving that the conjecture still held true for this type. After the interviewer's additional prompt asking them to examine (2) in Task 1-2, they recognised that Fig. 3e, f were local counterexamples to their initial proof and revised the proof.

The tasks implemented in this interview were thus shown to be helpful for facilitating the students' process of heuristic refutation. The condition of Task 1-1 is implicit in the sense that although the task includes a particular diagram, there is no specification regarding the positions of points A-D in the task sentences. This task condition led the students to proof construction for the given diagram and enabled them to produce the six types of diagrams, which had the potential to refute their conjecture, with the help of the dragging function of the DGE in Task 1-2. In our previous study (Komatsu et al., 2017), conversely, many secondary school students were found to encounter difficulties drawing diagrams that refuted their proofs in paper-and-pencil environments. The tasks used in that study were more difficult than those in case 1; nevertheless, without the use of dragging it would have been challenging for the three students in case 1 to produce various diagrams different from the one given in the original problem and thus to tackle their task also. Furthermore, the sequence of Tasks 1-1 and 1-2 was found to be beneficial for stimulating subsequent student activity. When discovering Fig. 3e, Sakura and Yuka felt a contradiction between the truth of their conjecture, as proved in Task 1-1, and the refutation in Task $1-2$, where they judged this type to be a counterexample to their conjecture. This contradiction triggered the debate with Kakeru, where Sakura and Yuka's judgement was revised by Kakeru's proof showing that their conjecture remained true in this type.

These effects of our task design are scrutinised and discussed in the next section through the analysis of the second case utilising different tasks.

\section{Case 2: Kenta and Tsubasa Tackle Task 2}

\section{The Proof and Types of Diagrams Produced by the Students}

In this section, we describe and analyse the interview for Task 2, in which a pair of undergraduates, Kenta and Tsubasa, participated. In Task 2-1, they initially planned to prove the statement by showing that the diagonals of quadrilateral AECF intersected at their midpoints, but ultimately abandoned their plan due to its difficulty. They then saw the possibility of proving the congruence of $\triangle \mathrm{ABE}$ and $\triangle \mathrm{CDF}$, and took the direction of 


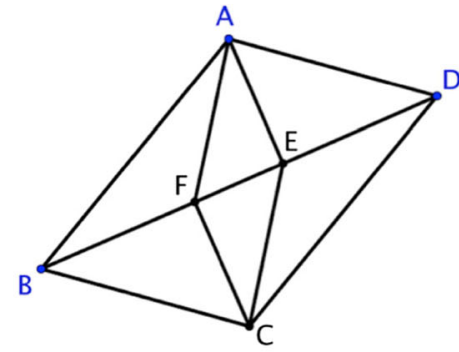

a

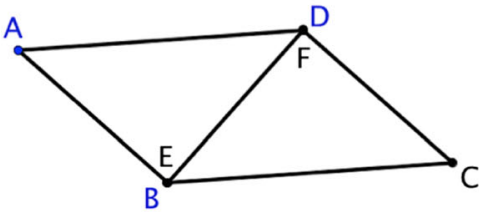

c

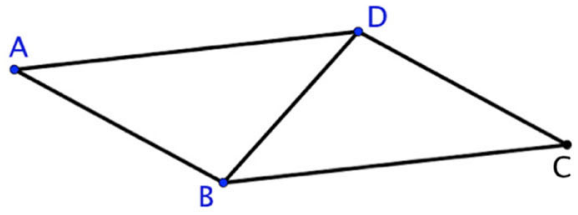

b

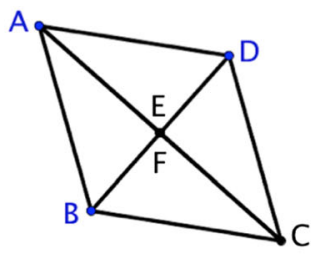

d

Fig. 4 The types of diagrams produced by the undergraduates in Task 2-2

showing that a pair of opposite sides of quadrilateral AECF were equal and parallel. Eventually, they completed the proof on their worksheet as summarised below:

$\angle \mathrm{AEB}=\angle \mathrm{CFD}=90^{\circ}$ and $\mathrm{AB}=\mathrm{CD}$ (the suppositions).

Since $\mathrm{AB} / / \mathrm{DC}$ (the supposition), $\angle \mathrm{ABE}=\angle \mathrm{CDF}$ (alternate angles).

Hence, $\triangle \mathrm{ABE} \equiv \triangle \mathrm{CDF}$ (a congruence condition for right-angled triangles), and thus $\mathrm{AE}=\mathrm{CF}$.

Since $\angle \mathrm{AEF}=\angle \mathrm{CFE}$ (the supposition), $\mathrm{AE} / / \mathrm{CF}$.

Hence, quadrilateral AECF is a parallelogram (a condition for parallelograms).

After this proof, Kenta and Tsubasa worked on Task 2-2 with the DGE and produced the four types of diagrams shown in Fig. 4. They first mentioned the case where the positions of points $\mathrm{E}$ and $\mathrm{F}$ were reversed (Fig. 4a). After that, they discovered the case where the perpendicular lines from points $\mathrm{A}$ and $\mathrm{C}$ disappeared because those lines did not intersect with diagonal BD (Fig. 4b). When exploring this case, the undergraduates produced a border case between Figs. 2 and $4 \mathrm{~b}$ where points $\mathrm{E}$ and $\mathrm{F}$ coincided with points $\mathrm{B}$ and D, respectively (Fig. 4c). They also produced the case where points E and F coincided (Fig. 4d).

The undergraduates considered that the statement in Task 2-1 is true for Fig. 4a, c. Regarding Fig. 4d, they said, "In this case, there is no quadrilateral [AECF] itself, so difficult". When the interviewer asked what kind of parallelogram ABCD led to this type, Tsubasa answered, "Rhombus". 4

\footnotetext{
${ }^{4}$ The undergraduates included squares as a subtype of rhombus based on their understanding of the hierarchical classification of quadrilaterals.
} 
In the analysis that follows, we focus on the undergraduates' engagement with the case of Fig. $4 \mathrm{~b}$.

\section{Extension of the Statement}

The following is the undergraduates' exchange when they discovered Fig. 4b:

153 Tsubasa: The case when [the statement is] not true .... Oh, this is different.

154 Kenta: Yes .... There is a case where we cannot draw the perpendicular lines to the diagonal.

155 Tsubasa: Well ....

156 Kenta: What figure?

157 Tsubasa: That is.

158 Kenta: The case where [the perpendicular lines are] outside [the parallelogram].

159 Tsubasa: Yes, it is.

160 Kenta: Yeah. This is a case when [the perpendicular lines] disappear.

161 Tsubasa: Well. After this [Fig. 4c].

162 Kenta: Yes. [The perpendicular lines] disappear.

163 Tsubasa: [The statement is] not true in this case.

This exchange confirms that the undergraduates encountered a contradiction between what they had obtained before their discovery of Fig. $4 b$, on the one hand, and the actual discovery, on the other. They had been convinced of the truth of the given statement by proving it in Task 2-1 and had increased their conviction at the beginning of Task $2-2$ by verifying that the statement was true for Fig. 4a. Nevertheless, the undergraduates subsequently discovered Fig. 4b, where the perpendicular lines from points $\mathrm{A}$ and $\mathrm{C}$ did not intersect with diagonal BD (line 154), and thus, the perpendicular lines disappeared in the DGE (lines 160 and 162). On this basis, Kenta and Tsubasa considered that this type was a counterexample to the statement in Task 2-1 (line 163). After that, they began to examine this type more deeply:

166 Kenta: Because [the perpendicular lines] disappear, we have to make them visible.

167 Tsubasa: Yes. We have to make [them] visible. 
Fig. 5 The extended case

168 Kenta: Does it mean changing [diagonal] BD to a line?

169 Tsubasa: Yes.

As both of the undergraduates stated "we have to" (lines 166 and 167), it is clear that the contradiction they encountered significantly stimulated them to extend the given statement so that Fig. $4 \mathrm{~b}$ could be considered. They engaged in this extension by drawing line $\mathrm{BD}$ and constructing perpendicular lines that passed through points $\mathrm{A}$ and $\mathrm{C}$ and intersected with line $\mathrm{BD}$ (Fig. 5).

\section{The Proof of the Extended Statement}

Tsubasa proposed to prove that in Fig. 5, quadrilateral AECF was also a parallelogram, and Kenta agreed with his proposal. Tsubasa retrospectively mentioned this proposal in the subsequent phase of the interview: "I supposed [quadrilateral AECF was] a parallelogram at first glance, but I couldn't be convinced".

After the undergraduates saw the possibility of showing the congruence of $\triangle \mathrm{ABE}$ and $\triangle \mathrm{CDF}$ in Fig. 5, Kenta suggested using the earlier proof they had produced in Task 2-1: "Well, how about a similar way to the previous one?" They found that it was possible to show in a similar way that segments $\mathrm{AE}$ and $\mathrm{CF}$ were parallel. Then, the undergraduates started to write a proof for Fig. 5, beginning by copying the earlier proof, but noticed that the equality of $\angle \mathrm{ABE}$ and $\angle \mathrm{CDF}$ could not be derived in the same way as before: ${ }^{5}$

251 Tsubasa: First of all, we have to show this parallel. AB parallel CD.

252 Kenta: We show the parallel. This is the same as previous.

255 Tsubasa: Next is, because alternate angles are equal, right?

266 Kenta: There $[\angle \mathrm{ABE}]$ and where?

267 Tsubasa: Here $[\angle \mathrm{ABE}]$ and here $[\angle \mathrm{CDF}]$.

\footnotetext{
${ }^{5}$ Some parts of the transcript are omitted in this excerpt as the full transcript is too long.
} 
274 Kenta: What? Are those alternate angles?

275 Tsubasa: Can't we say that?

276 Kenta: Alternate angles are ... inside and inside of parallel, right?

277 Tsubasa: I see. We can't say [that]. We must consider the supplement.

278 Kenta: Since $[\angle \mathrm{ABE}$ and $\angle \mathrm{CDF}$ are $]$ outside and outside.

279 Tsubasa: Well, we can make a subtraction from 180 .

Tsubasa first thought that the initial proof was applicable here, as he supposed that $\angle \mathrm{ABE}$ and $\angle \mathrm{CDF}$ were alternate angles (lines 255, 267 and 275). However, Kenta's objections (lines 266, 274 and 276) helped Tsubasa realise his mistake (line 277). Based on the equality of $\angle \mathrm{ABD}$ and $\angle \mathrm{CDB}$ (since $\mathrm{AB} / / \mathrm{CD}$ ), the undergraduates considered the supplements of these angles and showed that ' $\angle \mathrm{ABE}=180^{\circ}-\angle \mathrm{ABD}=180^{\circ}-\angle \mathrm{CDB}=\angle \mathrm{CDF}$ '. They used the initial proof to show the remaining parts and thus were able to prove that quadrilateral AECF is a parallelogram in the extended case in Fig. 5.

This was not the end of the students' discussion of the task; as captured in the next section, their discussion next turned to the issue of the definition of a mathematical object.

\section{Extension by Redefinition}

After the proof, Kenta retrospectively stated, "I realise that the meaning of the term 'diagonal' changes in various ways". When the interviewer asked the undergraduates about the definition of a diagonal, they answered as follows:

367 Kenta: Definition [laughing], um.

368 Tsubasa: I wonder how to define. Of course, because of this [pointing at the screen of the students' computer], it is not the inside [segment].

369 Kenta: Yeah, what we think this time is not the inside.

370 Tsubasa: It would be a kind of line.

371 Kenta: Perhaps. There is a polygon.

372 Tsubasa: Opposite sides. Opposite sides?

373 Kenta: Nonadjacent.

374 Tsubasa: Vertices.

375 Kenta: Yes, connecting two vertices.

376 Tsubasa: A line that is connecting. 
This exchange shows that the undergraduates extended the given statement not by altering 'diagonal BD' in the problem sentence in Task 2-1 to 'line BD', but rather by discarding the typical definition of 'diagonal', namely a segment connecting two nonadjacent vertices of a polygon, and redefining a diagonal as a line connecting such vertices (e.g. lines 370 and 376). We come back to this point in the 'Discussion' section because mathematical definitions are relevant to Lakatos's work and hence are an aspect of our research.

\section{Analysis of Case 2}

Similar to the secondary school students in case 1, the undergraduates in case 2 were able to work effectively on the mathematical activity shown in Fig. 1. They proved the given statement in Task 2-1, and then scrutinised whether it was always true. They then discovered Fig. 4b, which they admitted to be a counterexample to the statement. However, they extended the statement to include this type and proved the truth of the extended statement by retaining and modifying their earlier proof.

This process undertaken by the undergraduates was facilitated by the tasks they had tackled. Based on the particularity of the given diagram, Task 2-1 implicitly assumes that the perpendicular lines from points $\mathrm{A}$ and $\mathrm{C}$ intersect with diagonal $\mathrm{BD}$ and that quadrilateral AECF is constructed. This task condition engaged the undergraduates in proof construction for the given case, and enabled them to discover a refutation of the statement (as seen in Fig. 4b). Regarding Task 2-2, related to the use of the DGE, we previously implemented almost the same task in paper-and-pencil environments with secondary school students and undergraduate students: although many students easily found rhombuses and squares (as in Fig. 4d) as refutations of the statement, only a few were able to discover the case of Fig. $4 \mathrm{~b}$ by themselves. To succeed in this discovery, it is necessary to draw a parallelogram that is slanted considerably to the left-hand side, but this is difficult for students because such shape of parallelogram is rarely shown in textbooks and by teachers. In contrast, the DGE in our current study helped the undergraduates explore various shapes of parallelograms with its dragging function and thus independently discover Fig. 4b. Furthermore, the sequence of Tasks 2-1 and 22 was crucial in prompting the contradiction in which despite proving the statement, the undergraduates discovered Fig. $4 \mathrm{~b}$ to refute it. This contradiction triggered subsequent activity (as seen in lines 166 and 167 in the transcripts) where they spontaneously extended the statement to include Fig. $4 \mathrm{~b}$ (note that there was no indication of revision of the statement in Task 2-2, where only the truth of the statement was questioned).

\section{Discussion}

In our earlier studies, we aimed for paper-and-pencil task designs that enabled students to engage in mathematical activity involving proofs and refutations (e.g. Komatsu, 2017; Komatsu et al., 2017). The current study advances our research in two ways. First, we have transposed our investigations into DGEs to explore the impact of using DGE on the way that students produce counterexamples. Second, while previously we had clarified the characteristics of the tasks used, we had not specified principles underpinning the design of the tasks. In contrast, the present study develops such 
principles: using tasks whose conditions are purposefully implicit, providing tools that enhance the production of counterexamples, and increasing students' recognition of contradictions (see Table 1). As analysed in the previous subsections, we show how the tasks were designed according to the principles that we developed and how the designed tasks played a significant role in enabling the students to engage in heuristic refutation.

The contribution of our study thus consists in not only creating individual tasks but also theoretically developing, and empirically testing, design principles that underpin task creation. Task design principles are not always specified in mathematics education research; as Kieran et al. (2015) recently pointed out, "[d] espite the recent growth spurt of design studies within mathematics education, the specificity of the principles that inform task design in a precise way remains both underdeveloped and, even when somewhat developed, under-reported" (p. 74).

No doubt academic research and daily teaching practice have different contexts, and each classroom is also its own unique context; hence, teachers need to adjust tasks developed and used in research to reflect the contexts of their classes if they are to implement the tasks effectively and achieve the intended goals. To ease this adjustment, it is vital to make task design principles explicit, recognising, with Stylianides and Stylianides (2013), that "[k]nowledge of what counts as a 'theoretically essential component' of an intervention can support decisions about what should stay invariant, and what may be modified and how, when considering how the intervention can be adapted for use in diverse classroom contexts" (p. 338). Moreover, given that the number of tasks developed in each research study is usually quite limited (as is the case here), specifying design principles also has the advantage of allowing teachers and researchers to use the principles to create more tasks themselves.

In our research, the aforementioned theoretically essential components consist of the mathematical process depicted in Fig. 1 and our three task design principles shown in Table 1. In our interview for Task 1, the students were asked to make a conjecture and to scrutinise both the truth of their conjecture and the validity of their proof. However, not all of these actions are required by the mathematical process in Fig. 1 or by the design principles. Thus, if teachers are concerned about time constraints, they may modify Task 1-1 to present students the statement - the similarity of $\triangle \mathrm{PAB}$ and $\triangle \mathrm{PDC}$ - as a given one (like Task 2-1) or modify Task 1-2 to question only the truth of the conjecture/statement. Focusing on the validity of a proof would be another option, by which it would be possible to concentrate student activity on proof validation and modification (e.g. Alcock \& Weber, 2005; Komatsu et al., 2017; Selden \& Selden, 2003). Thus, the design principles developed in this study can provide teachers with a framework within which to conduct their own flexible task design.

Furthermore, our design principles are not conceptually restricted to geometry education, because the 'tools' mentioned in the second design principle are not only DGE tools. Hence, it may be possible to extend the principles to different areas; one possibility is algebra, another area where proofs are taught at the secondary school level. This is an issue for further research aimed towards introducing heuristic refutation in broader content areas.

Before concluding this paper, it would be worthwhile to refer to the undergraduates' behaviour regarding definition in case 2. Lakatos's research was deeply involved with the development of definitions (e.g. Kontorovich \& Zazkis, 2016; Larsen \& Zandieh, 2008; Ouvrier-Buffet, 2006). He described the history of the concepts of polyhedron 
and of uniform convergence of sequences of functions in terms of the development of definitions, for example through what he called 'monster barring' (ad hoc alteration of definitions to rule out problematic counterexamples) and proof-generated concepts. In case 2 in our study, the undergraduates redefined a diagonal as a line connecting two nonadjacent vertices of a polygon in order to extend the original statement in Task 2-1 for Fig. 4b. This redefinition increased the content of the original statement (Komatsu, 2016; Lakatos, 1976) in the sense that Fig. 4b, which had been initially regarded as a counterexample to the original statement, was now included as an example under the extended statement. However, there is a problem here: despite the undergraduates' success in the extension of the original statement, their redefinition is not compatible with the normal definition of diagonals, wherein diagonals are regarded as a kind of segment. The undergraduates could have performed such an extension without changing the standard definition if they had revised 'diagonal BD' in the problem sentence in Task 2-1 to 'line BD'. Thus, this paper reflects that the action of examining definitions is closely connected to the process of handling refutations.

\section{Conclusions}

Given the centrality of task design for enhancing student learning of mathematics, our research has explored task design for facilitating the development of heuristic refutation in DGEs. Although mathematical activity involving heuristic refutation is regarded as significant in school mathematics, much remains under-explored in mathematics education research about possible ways of facilitating students' success in this activity. Separately, although the affordances of using DGEs have been shown in the literature, task design in DGEs also remains under-studied. To address these issues, we theoretically developed task design principles based on the existing literature and empirically tested these principles by implementing and analysing task-based interviews. We have shown that using tasks designed from our principles can enable students to engage successfully in heuristic refutation.

There are limitations to the study reported in this paper, however. Given that the number of task-based interviews conducted was small, and the participants were relatively strong in mathematics, it is necessary to carry out additional task-based interviews with other types of students. It would also be worthwhile to unpack the findings of this study further and verify them in regular classroom settings. Another issue is in looking into ways in which students explore definitions during the process of handling refutations.

Acknowledgements We wish to express our thanks to Yasuyuki Sakamoto for his support for conducting our interview with his students, and to the anonymous reviewers for their helpful comments on earlier versions of this paper. Parts of earlier versions of this paper were presented at the 10th Congress of European Research in Mathematics Education (Dublin, Ireland, 2017). This study is supported by the Japan Society for the Promotion of Science (Nos. 15H05402, 16H02068 and 26282039).

Open Access This article is distributed under the terms of the Creative Commons Attribution 4.0 International License (http://creativecommons.org/licenses/by/4.0/), which permits unrestricted use, distribution, and reproduction in any medium, provided you give appropriate credit to the original author(s) and the source, provide a link to the Creative Commons license, and indicate if changes were made. 


\section{References}

Alcock, L., \& Weber, K. (2005). Proof validation in real analysis: Inferring and checking warrants. The Journal of Mathematical Behavior, 24(2), 125-134.

Arzarello, F., Olivero, F., Paola, D., \& Robutti, O. (2002). A cognitive analysis of dragging practises in Cabri environments. ZDM - The International Journal on Mathematics Education, 34(3), 66-72.

Baccaglini-Frank, A., Antonini, S., Leung, A., \& Mariotti, M. A. (2017). Designing non-constructability tasks in a dynamic geometry environment. In A. Leung \& A. Baccaglini-Frank (Eds.), Digital technologies in designing mathematics education tasks: Potential and pitfalls (pp. 99-120). New York: Springer.

Baccaglini-Frank, A., \& Mariotti, M. A. (2010). Generating conjectures in dynamic geometry: The maintaining dragging model. International Journal of Computers for Mathematical Learning, 15(3), 225-253.

Balacheff, N. (1991). Treatment of refutations: Aspects of the complexity of a constructivist approach to mathematics learning. In E. von Glasersfeld (Ed.), Radical constructivism in mathematics education (pp. 89-110). Dordrecht: Kluwer Academic Publishers.

de Villiers, M. (2004). The role and function of quasi-empirical methods in mathematics. Canadian Journal of Science, Mathematics, and Technology Education, 4(3), 397-418.

de Villiers, M. (2010). Experimentation and proof in mathematics. In G. Hanna, H. N. Jahnke, \& H. Pulte (Eds.), Explanation and proof in mathematics: Philosophical and educational perspectives (pp. 205221). New York: Springer.

D’Mello, S., Lehman, B., Pekrun, R., \& Graesser, A. (2014). Confusion can be beneficial for learning. Learning and Instruction, 29, 153-170.

Fahlgren, M., \& Brunström, M. (2014). A model for task design with focus on exploration, explanation, and generalization in a dynamic geometry environment. Technology, Knowledge and Learning, 19(3), 287315 .

Hadas, N., Hershkowitz, R., \& Schwarz, B. B. (2000). The role of contradiction and uncertainty in promoting the need to prove in dynamic geometry environments. Educational Studies in Mathematics, 44(1), 127150.

Hanna, G. (1995). Challenges to the importance of proof. For the Learning of Mathematics, 15(3), 42-49.

Hanna, G. (2000). Proof, explanation and exploration: An overview. Educational Studies in Mathematics, 44(1), 5-23.

Healy, L., \& Hoyles, C. (2000). A study of proof conception in algebra. Journal for Research in Mathematics Education, 31(4), 396-428.

Healy, L., \& Hoyles, C. (2001). Software tools for geometrical problem solving: Potentials and pitfalls. International Journal of Computers for Mathematical Learning, 6(3), 235-256.

Hollebrands, K., Laborde, C., \& Sträßer, R. (2008). Technology and the learning of geometry at the secondary level. In M. K. Heid \& G. W. Blume (Eds.), Research on technology and the teaching and learning of mathematics: Volume 1, research syntheses (pp. 155-205). Charlotte: Information Age Publishing.

Hoyles, C., \& Küchemann, D. (2002). Students' understandings of logical implication. Educational Studies in Mathematics, 51(3), 193-223.

Kieran, C., Doorman, M., \& Ohtani, M. (2015). Frameworks and principles for task design. In A. Watson \& M. Ohtani (Eds.), Task design in mathematics education: An ICMI study 22 (pp. 19-81). New York: Springer.

Ko, Y. Y., \& Knuth, E. J. (2013). Validating proofs and counterexamples across content domains: Practices of importance for mathematics majors. The Journal of Mathematical Behavior, 32(1), 20-35.

Komatsu, K. (2016). A framework for proofs and refutations in school mathematics: Increasing content by deductive guessing. Educational Studies in Mathematics, 92(2), 147-162.

Komatsu, K. (2017). Fostering empirical examination after proof construction in secondary school geometry. Educational Studies in Mathematics, 96(2), 129-144.

Komatsu, K., Jones, K., Ikeda, T., \& Narazaki, A. (2017). Proof validation and modification in secondary school geometry. The Journal of Mathematical Behavior, 47, 1-15.

Komatsu, K., Tsujiyama, Y., Sakamaki, A., \& Koike, N. (2014). Proof problems with diagrams: An opportunity for experiencing proofs and refutations. For the Learning of Mathematics, 34(1), 36-42.

Kontorovich, I., \& Zazkis, R. (2016). Turn vs. shape: Teachers cope with incompatible perspectives on angle. Educational Studies in Mathematics, 93(2), 223-243.

Laborde, C. (1995). Designing tasks for learning geometry in a computer-based environment. In L. Burton \& B. Jaworski (Eds.), Technology in mathematics teaching: A bridge between teaching and learning (pp. 35-67). London: Chartwell-Bratt. 
Lakatos, I. (1976). Proofs and refutations: The logic of mathematical discovery. Cambridge: Cambridge University Press.

Lakatos, I. (1978). Mathematics, science and epistemology: Philosophical papers (Vol. 2). Cambridge: Cambridge University Press.

Lampert, M. (1990). When the problem is not the question and the solution is not the answer: Mathematical knowing and teaching. American Educational Research Journal, 27(1), 29-63.

Larsen, S., \& Zandieh, M. (2008). Proofs and refutations in the undergraduate mathematics classroom. Educational Studies in Mathematics, 67(3), 205-216.

Leron, U., \& Zaslavsky, O. (2013). Generic proving: Reflections on scope and method. For the Learning of Mathematics, 33(3), 24-30.

Leung, A. (2011). An epistemic model of task design in dynamic geometry environment. ZDM - The International Journal on Mathematics Education, 43(3), 325-336.

Leung, A., \& Bolite-Frant, J. (2015). Designing mathematics tasks: The role of tools. In A. Watson \& M. Ohtani (Eds.), Task design in mathematics education: An ICMI study 22 (pp. 191-225). New York: Springer.

Lin, F. L., Yang, K. L., Lee, K. H., Tabach, M., \& Stylianides, G. (2012). Principles of task design for conjecturing and proving. In G. Hanna \& M. de Villiers (Eds.), Proof and proving in mathematics education: The 19th ICMI study (pp. 305-325). New York: Springer.

Marradez, R., \& Gutiérrez, Á. (2000). Proofs produced by secondary school students learning geometry in a dynamic computer environment. Educational Studies in Mathematics, 44(1), 87-125.

Olivero, F., \& Robutti, O. (2007). Measuring in dynamic geometry environments as a tool for conjecturing and proving. International Journal of Computers for Mathematical Learning, 12(2), 135-156.

Ouvrier-Buffet, C. (2006). Exploring mathematical definition construction processes. Educational Studies in Mathematics, 63(3), 259-282.

Polya, G. (1954). Induction and analogy in mathematics: Mathematics and plausible reasoning (Vol. 1). Princeton: Princeton University Press.

Prusak, N., Hershkowitz, R., \& Schwarz, B. B. (2012). From visual reasoning to logical necessity through argumentative design. Educational Studies in Mathematics, 79(1), 19-40.

Reid, D. A. (2002). Conjectures and refutations in grade 5 mathematics. Journal for Research in Mathematics Education, 33(1), 5-29.

Reid, D. A., Knipping, C., \& Crosby, M. (2008). Refutations and the logic of practice. In O. Figueras, J. L. Cortina, S. Alatorre, T. Rojano, \& A. Sepúlveda (Eds.), Proceedings of the Joint Meeting of PME 32 and PME-NA XXX (Vol. 4, pp. 169-176). Morelia, México: PME.

Selden, A., \& Selden, J. (2003). Validations of proofs considered as texts: Can undergraduates tell whether an argument proves a theorem? Journal for Research in Mathematics Education, 34(1), 4-36.

Sierpinska, A. (2004). Research in mathematics education through a keyhole: Task problematization. For the Learning of Mathematics, 24(2), 7-15.

Sinclair, M. P. (2003). Some implications of the results of a case study for the design of pre-constructed, dynamic geometry sketches and accompanying materials. Educational Studies in Mathematics, 52(3), 289-317.

Sinclair, N., Bartolini Bussi, M. G., de Villiers, M., Jones, K., Kortenkamp, U., Leung, A., \& Owens, K. (2016). Recent research on geometry education: An ICME-13 survey team report. ZDM - The International Journal on Mathematics Education, 48(5), 691-719.

Sinclair, N., \& Robutti, O. (2013). Technology and the role of proof: The case of dynamic geometry. In M. A. (. K.). Clements, A. Bishop, C. Keitel-Kreidt, J. Kilpatrick, F. K.-S. Leung (Eds.), Third international handbook of mathematics education (pp. 571-596). New York: Springer.

Stylianides, A. J., \& Stylianides, G. J. (2013). Seeking research-grounded solutions to problems of practice: Classroom-based interventions in mathematics education. ZDM - The International Journal on Mathematics Education, 45(3), 333-341.

Watson, A., \& Ohtani, M. (2015). Themes and issues in mathematics education concerning task design: Editorial introduction. In A. Watson \& M. Ohtani (Eds.), Task design in mathematics education: An ICMI study 22 (pp. 3-15). New York: Springer.

Zaslavsky, O. (2005). Seizing the opportunity to create uncertainty in learning mathematics. Educational Studies in Mathematics, 60(3), 297-321.

Zazkis, R., \& Chernoff, E. J. (2008). What makes a counterexample exemplary? Educational Studies in Mathematics, 68(3), 195-208. 